\title{
NOTA SOBRE LA PERCEPCION DEL CLIMA URBANO. EL EJEMPLO DE LA CIUDAD DE ZARAGOZA
}

\author{
Fernando LOPEZ MARTIN \\ Departamento de Geografía y Ordenación del Territorio \\ Universidad de Zaragoza
}

\begin{abstract}
Resumen: El artículo presenta los resultados del estudio empírico sobre la percepción del clima urbano en Zaragoza. Se describe la metodología y los resultados de la encuesta, que muestra que los habitantes de Zaragoza perciben la ciudad como más cálida que el campo y además los sábados y domingos como los días más lluviosos de la semana.
\end{abstract}

Palabras Clave: Clima urbano, percepción, Zaragoza.

\begin{abstract}
This work shows the results of the empirical analysis about the perception of the urban climate in Zaragoza. The results of the poll and methodology are described. It points out that the inhabitants of Zaragoza feel the city as a warmer place than the country. Moreover they belive that saturdays and sundays are more raing than the rest of the week.
\end{abstract}

Key words: Urban Climate, feel, Zaragoza.

\section{CLIMA PERCIBIDO Y CLIMA REAL}

La percepción del clima no ha sido aún tratada monográficamente en España, pero es evidente que la percepción climatológica y metereológica cómo los habitantes de un territorio perciben o se sienten el clima y el tiempo ha despertado el interés de diversos grupos de investigadores (geógrafos, urbanistas, psicólogos) desde los años sesenta en Estados Unidos, y en la década de los noventa en otras comunidades científicas, como la española (CALVO PALACIOS, 1976; MARTIN VIDE,1990)

De entrada, conviene sentar que el clima percibido puede diferir bastante de lo que un análisis climático objetivo, basado en series sistemáticas y homogéneas de 
datos meteorológicos correctamente registradas, establezca. Esto ha sido demostrado por un cierto número de trabajos basados en encuestas, sobre una base estadística a veces no muy amplia, pero con el aval de la rotundidad y coincidencia de sus resultados, que superan cualquier umbral de significación (DUNBAR, 1966; McBOYLE, 1972; FORUNIER, 1986; CHAMUSSY, 1988; etc). Aunque haya serias discrepancias entre el clima real y el percibido, el estudio de éste sigue teniendo interés climatológico, además de intrínsecamente psicológico y sociológico. Dos son, por lo menos, esos intereses climatológicos. Por una parte, el clima y el tiempo percibido suministran algunas de las pautas de las impresiones que despiertan en la población, y su comportamiento, determinados sucesos y hechos climáticos, en cuya discusión el climatólogo puede encontrar criterios útiles para el estudio del clima del pasado, antes del período de observaciones instrumentales. Por otra parte, lo que se perciba ha de orientar a los que transmiten y difunden la información climática, cada vez más numerosos, para que sus mensajes y expresiones sean los adecuados para una correcta interpretación y asimilación por parte del gran público.

La percepción climática tiene gran fuerza, por la procedencia de unas experiencias y vivencias personales, es una geografía subjetiva o personal (VILA VALENTI, 1983), consideradas, así dogma de fe individual. Casi nadie deja en un momento $\mathrm{u}$ otro, de expresar ciertas conclusiones sobre la evolución del tiempo atmosférico vivido. La fuerza que suele darse a estos juicios se explica, en efecto, por el hecho de que los argumentos proceden de la propia experiencia personal. Se oye decir, con frecuencia: "ahora no nieva tanto como antes", "ya no existe primavera", "el tiempo está loco", "ahora llueve menos", "antes hacía más frío". En algunos casos la afirmación puede ser cierta. Así, por ejemplo, en muchos centros de ciudades la frecuencia de los días de nieve o en que la nieve cuaja o de los días de helada ha disminuido apreciablemente a lo largo del siglo, con el crecimiento rápido de la ciudad y el reforzamiento del fenómeno de la "isla de calor" urbana. La intensidad que llega a alcanzar este fenómeno en las grandes ciudades (LOPEZ GOMEZ et al., 1988; MORENO GARCIA, 1990), junto con el alto porcentaje de la población asentado en ellas, hace que hoy un gran número de personas estén sometidas efectivamente a unas condiciones térmicas bastante más suaves que las que tuvieron hace unas décadas en el medio rural o en ciudades menores. Pero en muchos otros casos, percepción y realidad difieren. Así, al decir que antes hacía más frío se está, generalmente, afirmando que se notaba más que ahora, por unas condiciones de habitabilidad de las casas y de vestido más precarias, por ejemplo.

Climatológicamente, la memoria tiene un valor relativo, sus informaciones deben ser siempre tamizadas, filtradas con numerosas pruebas, confirmadas con otras muchas fuentes y, en el mejor de los casos, sustituidas por los datos registrados en los observatorios meteorológicos, cuando existen. La memoria es siempre selectiva, la realidad climatológica a escala humana, nunca, porque es el resultado de muchas 
pequeñas fluctuaciones, que, sólo en algunos casos, marcan una verdadera señal climática, siempre suave, a la escala citada. La memoria además, como es bien conocido, tiende a realizar una selección irregular: olvida o, en sentido contrario, magnifica ciertos hechos pasados, por el concurso de otras muchas circunstancias, y agranda y detalla lo más reciente, con un calendario propio de cada sujeto. No hay, pues, un seguro y fijo factor de conversión que transforme sus recuerdos en información con carácter climatológico, que, recuérdese, ha se ser homogénea, continua en el tiempo, registrada de un modo sistemático y, sobre todo, objetiva. Este último requisito no puede atribuirse a la percepción individual, pero es que incluso tampoco el de su continuidad en el tiempo ya que son, casi siempre, mayores las lagunas mentales, que las existentes en los archivos meteorológicos. Además, cada individuo, aun siguiendo pautas similares, tiene su propia sensibilidad ante los hechos atmosféricos, reparando más o menos en ellos, y de esta manera, haciendo inviable la comparación numérica entre las experiencias recordadas por varios sujetos.

A todo este complejo abanico de rasgos de la percepción climatológica hay que añadir los derivados del profundo cambio de modos de vida, de lugar de residencia y de situación social general experimentado por nuestras sociedades en el siglo $X X$, que ha variado patrones y referencias, hitos y valores de una manera extraordinaria. La percepción y los recuerdos meteorológicos habrán sufrido, con toda seguridad, alguna alteración por causa de esos profundos cambios.

Sin embargo a pesar de la complejidad de la percepción climatológica y meteorológica y de sus discrepancias con la realidad correspondiente, lo percibido tiene un gran valor, que el climatólogo no puede despachar sin una lectura, atenta, al menos en los dos temas apuntados al comienzo de este apartado. Pero son otras instancias, sociales, técnicos y autoridades, las que más han de prestar atención a esas informaciones subjetivas. Esto es así, porque los individuos se comportan en gran medida tal como perciben la realidad. Ejemplificando, no hará frío, según un dato térmico, en una determinada jornada, pero si la población percibe lo contrario, vestirá ropas de abrigo y quizás salga en menor número a las calles. Señalemos que bastaría distinguir la mencionada jornada con el carácter de electoral para que las repercusiones de tal percepción tengan sonada resonancia.

Nótese, de todo lo dicho, que se están tratando sólo algunos aspectos de la percepción (psicológica). Descartamos aquí el tema de las sensaciones y del confort o disconfort meteorológico y climático, cuyo estudio es propio de la Bioclimatología, y que puede esclarecerse a la luz de los balances energéticos.

Otra cuestión más compleja, pero no por ello menos importante, es la de la influencia que en la percepción climatológica y meteorológica individual, no ya sólo en la opinión pública, tienen los medios de comunicación. Hoy, cuando éstos hablan con gran profusión, y no siempre con fundamento, del cambio climático hacia unas 
temperaturas más altas, el gran público tiene asumido, y lo "percibe", en gran proporción, tal presunto cambio. Quizás algunas experiencias lejanas vividas vayan en el mismo sentido que el cambio climático anunciado, reforzando, de esta manera, su impresión. Es por ello por lo que deberá, en el estudio de la percepción climática, tenerse muy presente la "contaminación" que puedan sufrir los individuos por influencias externas informativas, $\mathrm{o}$, mejor, desinformativas, fundamentalmente.

\section{PERCEPCION DEL CLIMA URBANO}

El medio urbano supone una modificación tan importante de las condiciones naturales de la región o comarca en que se inserta, que sus habitantes quedan, por decirlo de algún modo, aislados de la realidad natural. Los viviendas presentan unas condiciones ambientales próximas a las del confort climático standard. Sea invierno o verano, se intenta mantener una temperatura siempre grata. La topografía urbana reduce fuertemente el Sky Factor Vision (es decir, el factor de visión del cielo, tan importante en los estudios de "isla de calor"), de modo que el urbanita apenas ve o repara en los cambios de estado del cielo, que son seguidos con la máxima atención por el hombre que reside en el medio rural, dadas las repercusiones que en ocasiones puede tener en sus actividades económicas. Todo ello causa el aislamiento aludido. Más, si cabe, por el hecho de que en muchos casos, aunque exceptuando un cierto porcentaje significativo de personas y actividades, el que llueva o no, en una ciudad, en una determinada jornada laboral escasa tiene repercusiones para un gran número de actividades y los trabajadores que las realizan. Pongamos el ejemplo de los empleados en oficinas, a menudo encerrados en espacios sin iluminación natural, aislados, pues, visual y acústicamente del medio atmosférico. Tan eficaz resulta ese aislamiento que para un elevado número de personas residentes en las ciudades resulta muy difícil responder, al término de una jornada, si llovió o no en ella. Y ello no sólo porque no repararon en ello, sino por la escasa incidencia que tiene en su ritmo cotidiano de actividades. De todas maneras hemos dicho que hay excepciones significativas, como, por ejemplo, taxistas y demás conductores, para los que la precipitación causa alteraciones importantes de su ritmo de trabajo y de actividad, no sólo por el fenómeno en sí, sino especialmente por las repercusiones que ocasiona en el medio urbano (congestiones de tráfico, etc..).

Sin embargo, esa apatía y desinterés mayoritario por el tiempo entre la población urbana, al margen de las repetidas frases con contenido meteorológico que completan un saludo convencional o permiten el inicio de una conversación, se torna en vivo interés de cara al fin de semana. Ello se explica, sin duda, por el hecho de que las actividades de ocio de esa población, realizadas hoy, y más en nuestros agradables climas, al aire libre en muchos casos, buscando la evasión física del medio urbano, en mayor grado cuanto mayor sea el nivel socieconómico, pueden quedar seriamente 
afectadas por el mal tiempo. $\mathrm{O}$, en todo caso, aun sin ser un impedimento para su realización, el mal tiempo limita, precisamente, las actividades más deseadas y gratas.

Este hecho, distorsiona extraordinariamente la percepción meteorológica y climatológica del hombre urbano, dando lugar a pulsaciones con ciclo semanal, como se pretende ver a continuación, analizando como perciben el clima los habitantes de Zaragoza.

\section{METODOLOGIA}

Después de vistas las posibilidades que ofrece el estudio de la percepción del clima urbano, se ha pretendido analizar el caso concreto de la ciudad de Zaragoza utilizando la metodología de CEHAK (1982) que se apoya en la encuesta directa al ciudadano, sobre su percepción del clima urbano, con preguntas realizadas en lenguaje sencillo y de forma directa, para ser contestadas por cualquier persona. Esta metodología ha sido validada en los estudios de MARTIN VIDE (1990), para la ciudad de Barcelona, por lo que intentamos en este trabajo aplicarlo con las garantías que esto supone.

Dos son los puntos básicos que centran el trabajo:

a)Averiguar si la población percibe alguna variación climática entre el campo y la ciudad, ya que es el paso previo para analizar el fenómeno climatológico urbano, ya que en las ciudades se produce uno de los fenómenos más definitorios del clima urbano, como es la formación de la "isla de calor". No es motivo de este trabajo hablar de este fenómeno investigado en todas sus vertientes por investigadores extranjeros desde hace más de 30 años y más recientemente en España por equipos de Geógrafos de las ciudades de Madrid, Barcelona, Valencia, Logroño, Sta. Cruz de Tenerife, Bilbao y Zaragoza que han dado fruto a varias Tesis Doctorales y al menos a tres libros dedicados al tema. Pero al plantearse este trabajo de investigación pensé que sería bueno comprobar, dentro de la percepción climática de los habitantes de la ciudad de Zaragoza, entre el campo y la ciudad, cual era su apreciación respecto al fenómeno de la temperatura. Este aspecto lo hemos analizado planteando dos preguntas a los encuestados:

1.- ¿Dónde cree que llueve más, en el campo o en la ciudad?.

2.- ¿Dónde cree que es mayor la temperatura, en el campo o en la ciudad?. 
b)Hemos planteado una cuestión para el análisis de la percepción del clima urbano en el aspecto concreto de las precipitaciones, formulando la siguiente pregunta:

1.- ¿Que día de la semana llueve más en la ciudad?.

Estas preguntas que han servido de base a esta investigación han formado parte de una encuesta, realizada por el propio autor, durante el mes de mayo de 1993 en la ciudad de Zaragoza a un universo de 200 personas entre los once y los ochenta años y desglosados por sexos con distintos niveles educativos, no pudiendo equilibrar la encuesta por lo que hay cierto predominio del grupo, entre 11-20 años, de sexo femenino y estudiantes de BUP que se traslucirá en la contestación a las preguntas.

\section{RESULTADOS}

Cuando se realizan estudios sobre el clima de las ciudades y su posible repercusión en los problemas del medio ambiente urbano, se parte en la mayor parte de los casos de la comparación de los datos de los observatorios meteorológicos situados en los cascos urbanos con los situados en la periferia urbana, para observar si existe una influencia directa de la aglomeración urbana en el clima de estas áreas.

Sabemos que efectivamente las aglomeraciones urbanas suponen numerosas modificaciones medioambientales y notables alteraciones climáticas, como señalan detalladamente OKE y HOBBS (1980), LOPEZ GOMEZ (1988), etc, como el aumento de las temperaturas y las precipitaciones en las ciudades respecto al campo, o la disminución de la humedad relativa.

Por esta razón el punto de partida del trabajo es el análisis de percepción, para comprobar que es lo que realmente sienten los habitantes de la ciudad de Zaragoza respecto a este tema, interrogándoles con dos preguntas, cuyos resultados analizamos a continuación.

\section{a) ¿Dónde cree que llueve más en el campo o en la ciudad?}

La formulación de esta cuestión, tenía la clara intención de poner de manifiesto dos hechos hipotéticos; el primero es que la población tiene especial sensibilidad respecto a las lluvias en el medio rural cuando se comparan con las de la ciudad, y en segundo lugar el desconocimiento sobre el fenómeno del clima urbano de los habitantes de la ciudad de Zaragoza. 
La respuestas de los 200 encuestados confirmaron la hipótesis, ya que el 53\% (106) casos respondieron que las lluvias eran mayores en el campo, 36,5\% (73 casos) que en la ciudad, $5 \%$ (10 casos) respondieron que por igual y 5,5\% (11 casos) no contestan.

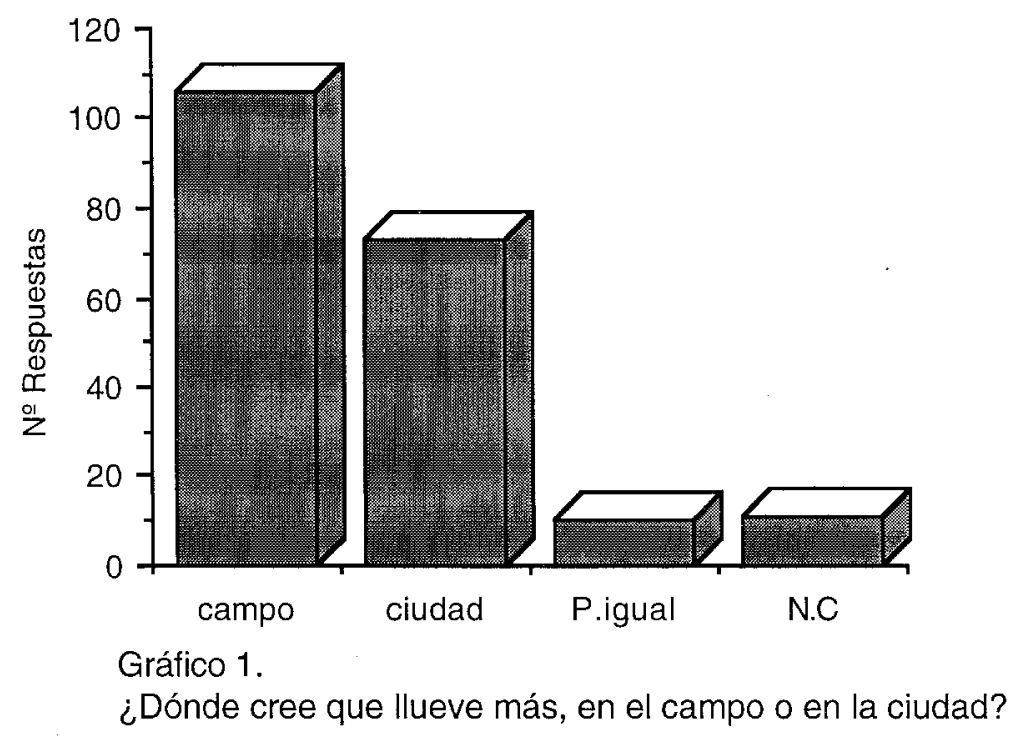

Se observa que los encuestados perciben que las lluvias se producen en mayor cantidad en el campo, una percepción totalmente errónea, ya que es totalmente contrastado que en la ciudad se producen entre un 10\%-15\% de precipitaciones más que en el medio rural debido entre otras causas a la mayor abundancia de polvo en suspensión (núcleos higroscópicos), inestabilidades térmicas, rugosidad de la edificación, etc.

Interrogados por las causas por las que percibian este predominio de las precipitaciones en el campo, casi todas las respuestas están asociadas con la concepción de que en el medio rural es donde se encuentran los ríos, embalses, donde se cultiva la tierra, donde el agua produce inundaciones y que por lo tanto debe llover más. Nos parece, que estas contestaciones sencillas y erróneas en cuanto a la percepción están directamente relacionadas con la facilidad que encuentra el agua para evacuarse en las ciudades (red alcantarillado) por lo que hay una menor percepción del fenómeno de la precipitación respecto a la ciudad, condicionando una falsa consideración del fenómeno. 
No podemos olvidar en este atrevido análisis de causas de este error de percepción, el sentimiento de indefensión manifestado por muchos encuestados, ante el fenómeno meteorológico de la precipitación en el campo, que en la mayoría de los casos no permite un refugio fácil, al contrario de lo que ocurre en esta misma situación en la ciudad. Por lo que la percepción del fenómeno es mucho mayor en el campo que en la ciudad.

En el análisis de esta pregunta por sexos, los resultados muestran una mayor inclinación de los hombres $(67,8 \%)$ al error de percepción analizado, frente a una igualdad en las mujeres ( $46 \%$ ) que responden por igual que las precipitaciones, mayores sean en el campo o en la ciudad.

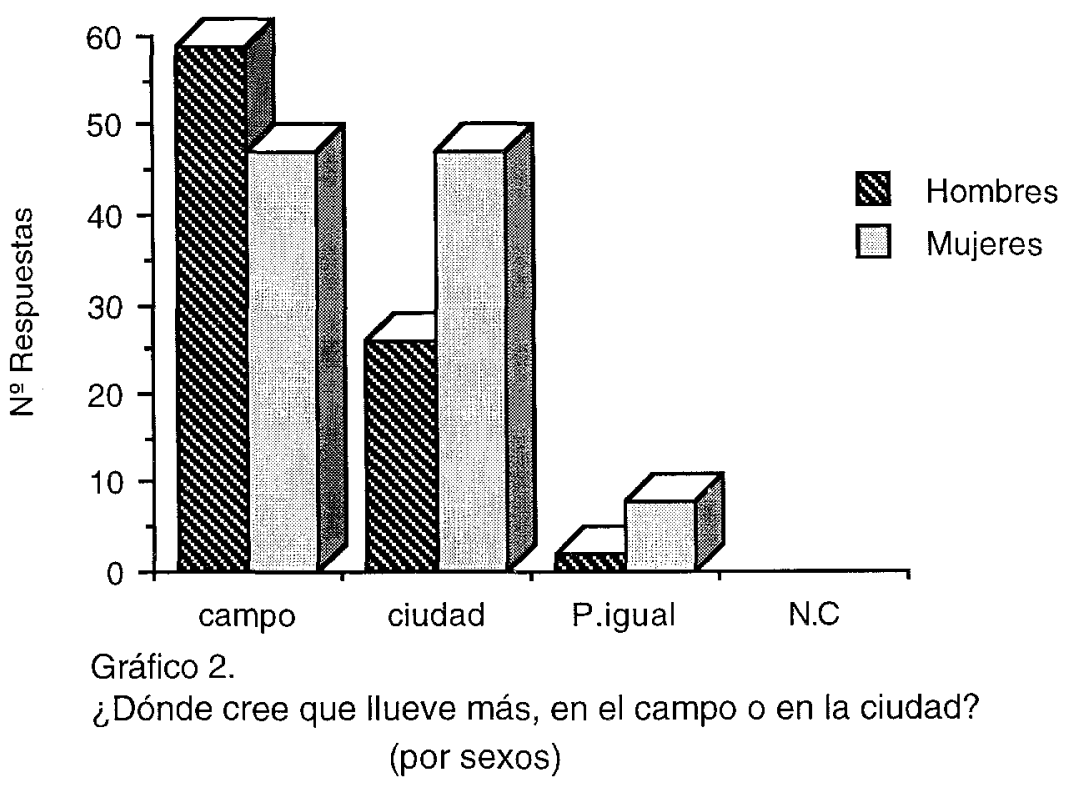

Los resultados ofrecidos muestran que en el caso de los hombres está marcada la percepción del medio rural como la zona con más precipitaciones, por todas las causas antes apuntadas.

En las mujeres se produce un equilibrio en las respuestas, pero en ninguno de los casos se apuntan razones correctas sobre el fenómeno, sino que simplemente es una contestación según sus sentimientos ante la pregunta realizada, por lo que no encontramos razones especiales a las ya señaladas en general para ambos sexos.

Por último habría que añadir como hecho importante en la percepción de las precipitaciones entre el campo y la ciudad, que Zaragoza capital tiene unas 
precipitaciones medias de $340 \mathrm{~mm} /$ año, que a pesar de suponer un 10\%-15\% más que en el medio rural circundante (Monegros y zona central del valle del Ebro) es la zona más seca de la Comunidad Autónoma y por tanto la percepción de los más de 600.000 hab. de Zaragoza es que el campo es mucho más húmedo, extrapolando el ámbito por el que se les interrogaba que era el campo próximo a la ciudad a todo el medio rural. Un puro proceso de percepción mental.

\section{b) ¿Dónde cree que es mayor la temperatura, en el campo o en la ciudad?}

Es este caso el resultado de la encuesta realizada con este fin arrojó unos resultados mucho más acordes con la realidad. El 72,5\% (145 casos) respondieron que la temperatura era mayor en la ciudad, 21\% (42 casos) dijeron que era mayor en el campo, un $1 \%$ ( 2 casos) que por igual y un $5,5 \%$ (11 casos) no contestaron.

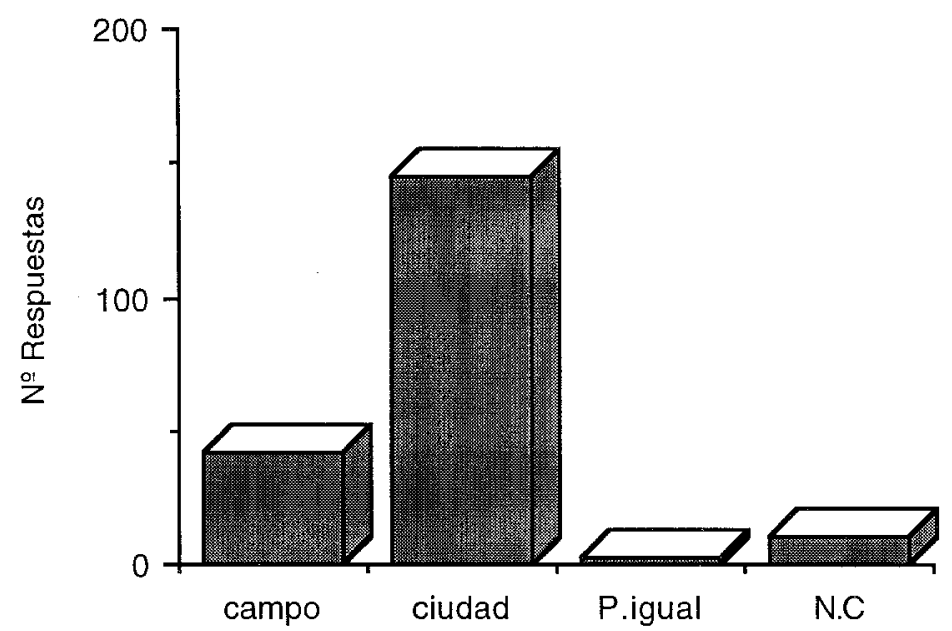

Gráfico 3.

¿dónde cree que es mayor la temperatura, en el campo o en la ciudad?

La percepción de los habitantes de Zaragoza respecto a las temperaturas que se producen en la ciudad y el campo circundante, señala bien a las claras, el efecto que se produce a partir del mes de mayo (fecha de la encuesta), cuando se empiezan a sufrir en esta ciudad, los rigores de la acumulación de calor en sus calles, además de que en este caso concreto se hubieran sucedido cuatro días de temperaturas muy altas, poco habituales en estas fechas en la capital de Ebro, dando así una percepción de mayor calor en la ciudad. 
Varios de los encuestados hacen referencia al dato de que "en el campo por la noche refresca y en la ciudad no" lógicamente muy relacionado con el fenómeno de la "isla de calor", a pesar que sabemos perfectamente que ésta es mucho más intensa en las situaciones anticiclónicas de los meses de invierno, hecho que también intuyen los habitantes de la ciudad de Zaragoza al señalar que en invierno hace más frío en el campo que en la ciudad y las heladas son mucho más frecuentes en los alrededores de la ciudad.

Esta cuestión desglosada por sexos, no presenta diferencias en las contestaciones por porcentajes, distinguiéndose las cifras en la gráfica por haber encuestado más mujeres que hombres: Hombres $77,1 \%$ (67 casos) ciudad, 22,9\% (20 casos) en el campo y en las mujeres $77 \%$ ( 78 casos) ciudad y $21 \%$ (22 casos) en el campo 1,8\% (2 casos) que por igual.

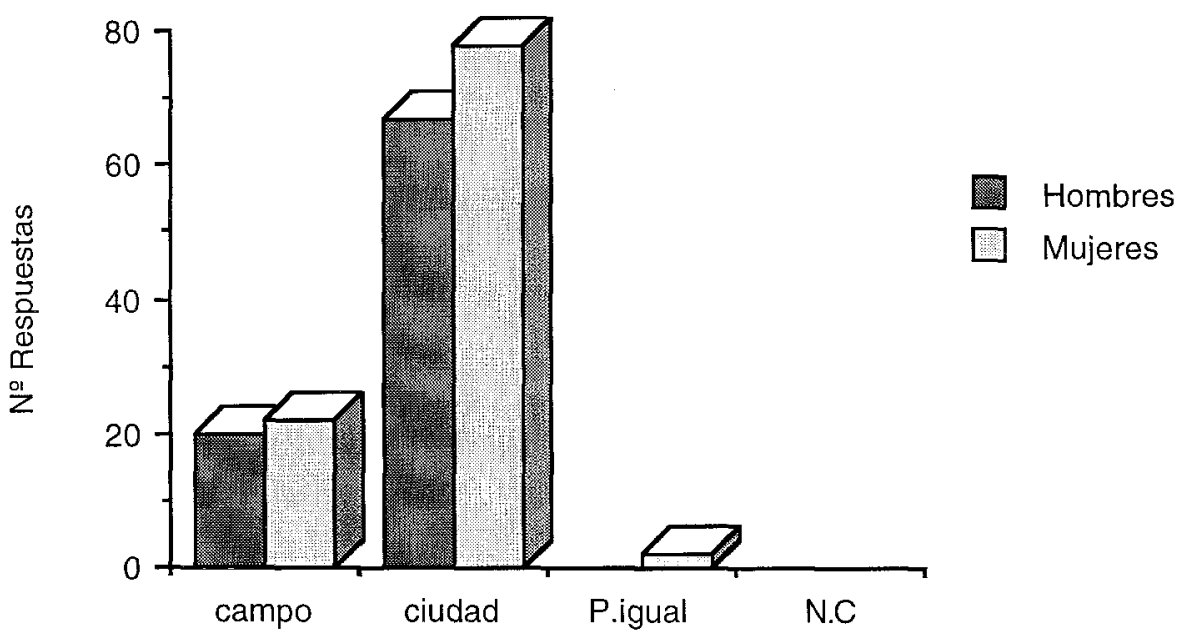

Gráfico 4.

¿Dónde cree que es mayor la temperatura, en el campo o en la ciudad? (por sexos)

Con el análisis de las dos cuestiones referidas a la percepción del clima de la ciudad de Zaragoza y su campo circundante, hemos podido comprobar como existen formas de percibir diferentes, respecto a los fenómenos meteorológicos de la precipitación y la temperatura, incluso percepciones erróneas respecto a la realidad científica, no dejando de ser muy interesante lo que sienten los ciudadanos ya que es lo que ellos sienten desde el punto de vista climatológico.

Siguiendo esta vía de investigación, creíamos que había que estudiar también que era lo que percibían los habitantes de Zaragoza respecto al clima concreto dentro 
de la ciudad, y para ello hemos utilizado una pregunta que ya han utilizado CEHAK (1982) y MARTIN VIDE (1990) en estudios anteriores, con resultados similares a los de la capital aragonesa.

\section{c) ¿Qué día de la semana llueve más en la ciudad?}

La realización de esta pregunta se efectuó igual que las anteriores a 200 personas durante el mes de mayo de 1993.

\begin{tabular}{|l|c|c|c|}
\hline \multicolumn{3}{|c|}{ DATOS ¿QUEDIADE LA SEMANA LLUEYEMAS EN LA CIUDAD? } \\
\hline Día de la semana & Hombres & Mujeres & Total \\
\hline Lunes & 21 & 17 & 38 \\
\hline Martes & 8 & 10 & 18 \\
\hline Mércoles & 5 & 8 & 13 \\
\hline Jueves & 5 & 6 & 11 \\
\hline Yiemes & 18 & 15 & 33 \\
\hline Sábado & 17 & 21 & 38 \\
\hline Domingo & 7 & 17 & 24 \\
\hline Porigual & 7 & 7 & 14 \\
\hline No contesta & 0 & 0 & 11 \\
\hline
\end{tabular}

Como se aprecia en el cuadro sólo el $7 \%$ (14 casos) del total contestaron que por igual, y un 5,5\% (1 caso) no contestaron. El resto eligió algún día de la semana. Así sobre el total el 19\% (38 casos) citó el sábado, el 16,5\% (33 casos) el viernes y el 12\% (24 casos) el domingo, ofreciendo el resto de días de la semana porcentajes inferiores al $9 \%$. Apreciándose un incremento brusco del viernes y sábado, con disminución el domingo (entre estos 3 días 47,5 de las respuestas dicen que es el día más lluvioso en la ciudad) y un incremento en el lunes (19\%) para disminuir nuevamente hasta el viernes. 


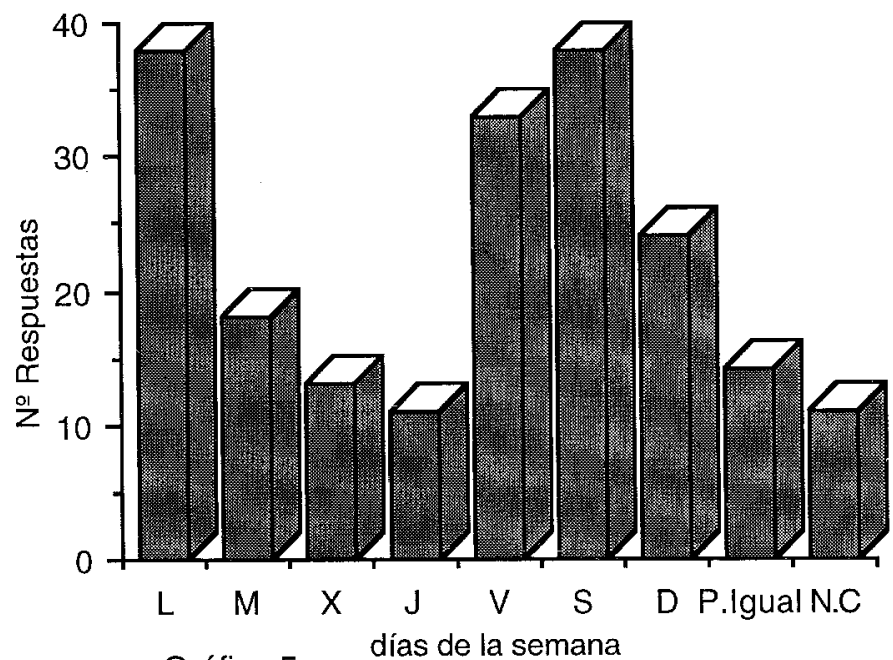

Gráfico 5.

¿Qué día de la semana llueve más en la ciudad?

La encuesta que arroja estos datos se realizó entre personas de sexos, edades y niveles de estudios que no han ofrecido especiales influencias sobre los resultados.

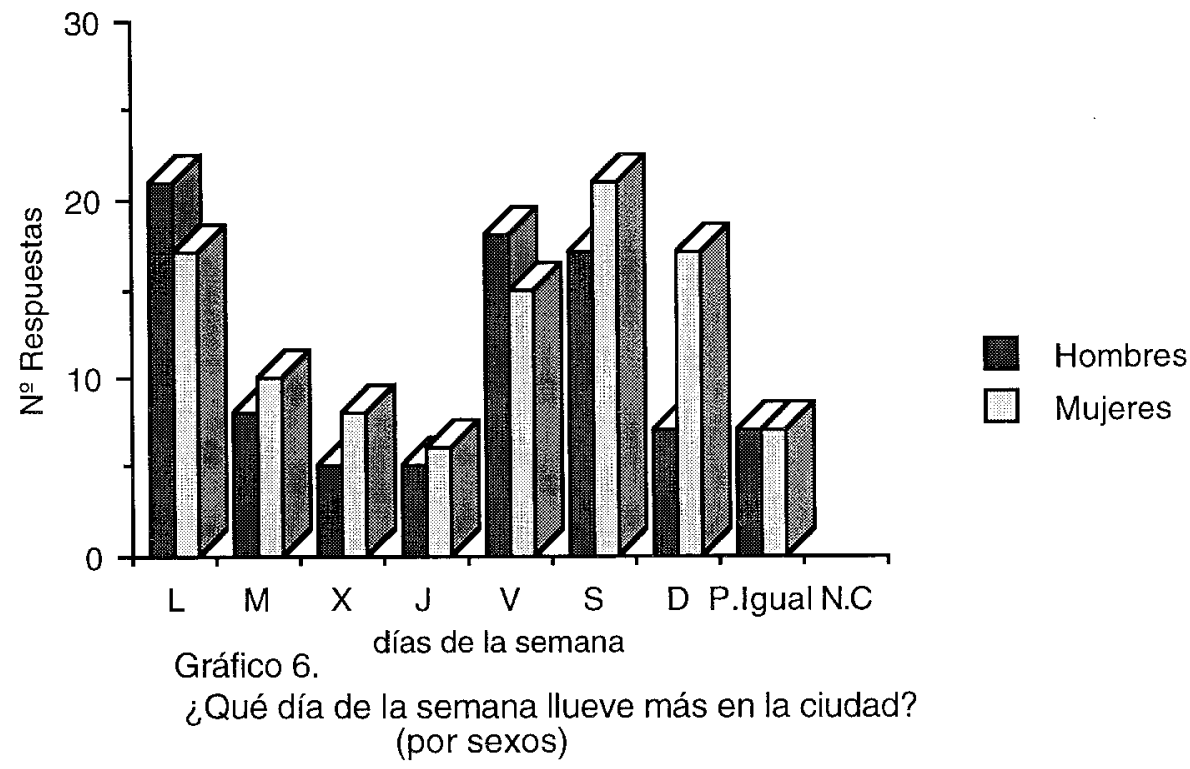


En el mes de mayo en la ciudad de Zaragoza y sobre todo a finales del mes de abril, se constata un período de buen tiempo con pocas precipitaciones, que en este caso concreto fueron más frecuentes los días martes y jueves.

Ante este hecho objetivo, ¿cómo es posible una percepción del clima urbano de Zaragoza tan errónea?. Tiene mucho que ver con fenómenos sociales, psicológicos y de moda, que sólo nos atreveremos a apuntar.

El habitante de la ciudad está especialmente sensibilizado ante el tiempo de últimos día de la semana, se nota que es mayor el disgusto ante el mal tiempo del fin de semana, ello deriva sin duda de la sociedad de ocio que necesita este período de asueto laboral para la expansión, por lo que se prefiere buen tiempo, ya que en la sociedad española la mayor parte de las actividades se realizan al aire libre.

En el caso concreto de Zaragoza y de este trabajo, se aprecia, por la abundancia de personas entre 14 y 17 años (estudiantes de BUP y COU) una mayor percepción de días lluviosos los viernes y sábados, días tradicionales de las salidas juveniles en nuestra ciudad, con una disminución el domingo, en el que se sale muy escasamente respecto a los días anteriores, para volver a subir el lunes, días de retorno a los colegios.

Esta modificación que ofrecen los jóvenes de la ciudad de Zaragoza es mínima, sólo significativa en la disminución de los domingos, tomando el mayor porcentaje de días lluviosos como algo negativo, ya que se prefieren días soleados y "buenos". A lo que hay que añadir lo que señala el Prof. Martín Vide "estamos en una sociedad con una moda heliófila", en la que, a principios de primavera se necesitan días forzosamente soleados para alcanzar un buen grado de "moreno", que es algo muy apreciado socialmente, por lo que se tiene especial sensibilidad sobre aquellos días en los que se pueden realizar estas actividades.

\section{DISCUSION}

Visto los resultados de la encuesta nos queda por último ver que grado de veracidad puede tener esta percepción del clima urbano. Lo cierto es que poca, ya que la mayoría de las investigaciones realizadas arrojan datos en los que se indica que las precipitaciones, tanto en frecuencia como en cantidad, suelen ser mayores en los días centrales de la semana que los sábados y domingos, debido a que la mayor actividad urbana e industrial de los días laborables de la semana, con un efecto, incluso, acumulativo desde los iniciales, potencia ciertos mecanismos de convección, además del incremento de partículas y núcleos de condensación lo que, a escala climatológica, 
se traduciría en una mayor frecuencia de precipitaciones en esos días. Lo que sería un fenómeno de modificación climática por causa urbana, es decir, específico de la climatología urbana. $Y$, en efecto, varias investigaciones sobre importantes ciudades y áreas urbanas americanas y europeas demuestran esta hipótesis. Por el contrario MORENO GARCIA (1988) afirma en el caso de Barcelona que la frecuencia de la precipitación es prácticamente equiprobable entre todos los días de la semana, no siendo cierto, por tanto, la creencia popular de los fines de semana lluviosos, ni las hipótesis verificada en grandes ciudades americanas y europeas. Hipótesis de Moreno, que es apoyada por el análisis diario de las precipitaciones de la ciudad de Zaragoza que presentan igualmente una equidistribución de las precipitaciones en todos los días de la semana, lo que indica bien a las claras que hay que profundizar más, en la investigación de estos temas.

Es evidente que el estudio de la percepción del clima urbano, tiene interés para el análisis del clima histórico, así como para analizar pautas de comportamiento de la población, ya que los habitantes se comportan como perciben, y la gente es elemento fundamental del medio ambiente urbano.

\section{BIBLIOGRAFIA}

CALVO PALACIOS, J.L. (1976): Apc tación metodológica al estudio geográfico del microclima urbano. Boletín de la Real Sociedad Geográfica. Madrid.

CAPEL,H. (1973): Percepción del medio y comportamiento geográfico, Revista de Geografía,VII,1 y 2, 58-150, Departamento de Geografía, Universidad de Barcelona

CEHAK,K.(1982): Note on the dependence of precipitation on the day of the week in a medium industrialized city, Archives for Meteorology, Geophysics and Bioclimatology, series B, 30 (3) 247-251, Viena.

CUADRAT, J.M., DE LA RIVA, J., LOPEZ MARTIN, F. y MARTI, A (1993): La ciudad y el clima, "la isla de calor" de Zaragoza. Actas del III Congreso de Ciudades Saludables. Ayto. de Zaragoza

CUADRAT, J. M., DE LA RIVA, J., LOPEZ MARTIN, F. y MARTI, A. (1993): El medio ambiente urbano en Zaragoza. Observaciones sobre "la isla de calor". Anales de Geografía de la Universidad Complutense, 13. Madrid.

HOBBS, J.E. (1980): Applied climatology. Westview, Colorado.

LOPEZ GOMEZ, A. et al. (1988): El clima urbano de Madrid: la isla de calor. Instituto de Economía y Geografía Aplicadas, C.S.I.C., Madrid. 
LOPEZ GOMEZ, A. et al. (1993): El clima de las ciudades españolas. Cátedra. Madrid.

LOPEZ GOMEZ, A. tal., (1993): El clima urbano de Madrid: teledetección de la isla de calor, M.O.P.T. Madrid.

MARTIN VIDE, J. (1990) La percepción del clima en las ciudades. Revista de Geografía XXIV. Universidad de Barcelona

MORENO GARCIA, Ma C. (1988): Frecuencias de la precipitación según los días de la semana en Barcelona, Revista de Geografía, XXI-XXII, 5-10, Departamento de Geografía, Universidad de Barcelona.

MORENO GARCIA, Ma C. (1990): Estudio del clima urbano de Barcelona: la "isla de calor". Departamento de Geografía Física y Análisis Geográfico Regional, Universidad de Barcelona (Tesis Doctoral).

OKE, T.R. (1980): Climatic impacts of urbanisation. Workshop Münster, Germany.

VILA VALENTI, J (1983): Introducción al estudio teórico de la Geografía. Barcelona, Ariel. 